\title{
THE PITUITARY GLAND AND IMPLANTATION IN THE TAMMAR WALLABY, MACROPUS EUGENII
}

\author{
J. P. HEARN* \\ Zoology Department, Australian National University, \\ Canberra, Australia
}

INTRODUCTION

Marsupial reproductive patterns include several unique features which suggest that true viviparity evolved independently in the Marsupialia and the Eutheria (Sharman, Calaby \& Poole, 1966; Tyndale-Biscoe, 1973). For example, the gestation period is generally similar in length to the oestrous cycle or shorter than it, there is prolonged extra-uterine development in the marsupium and embryonic diapause is a regular feature of many species. Comparison of the controlling mechanisms of these aspects of marsupial reproduction with those of eutherian reproductive patterns reveals the different ways in which the two groups have adapted to similar environmental problems.

The female tammar wallaby is a precise seasonal breeder. In Australia young are usually born in late January or early February, having developed from a quiescent blastocyst carried in the uterus from the previous year. The annual reproductive cycle is shown in Text-fig. 1. After birth the young

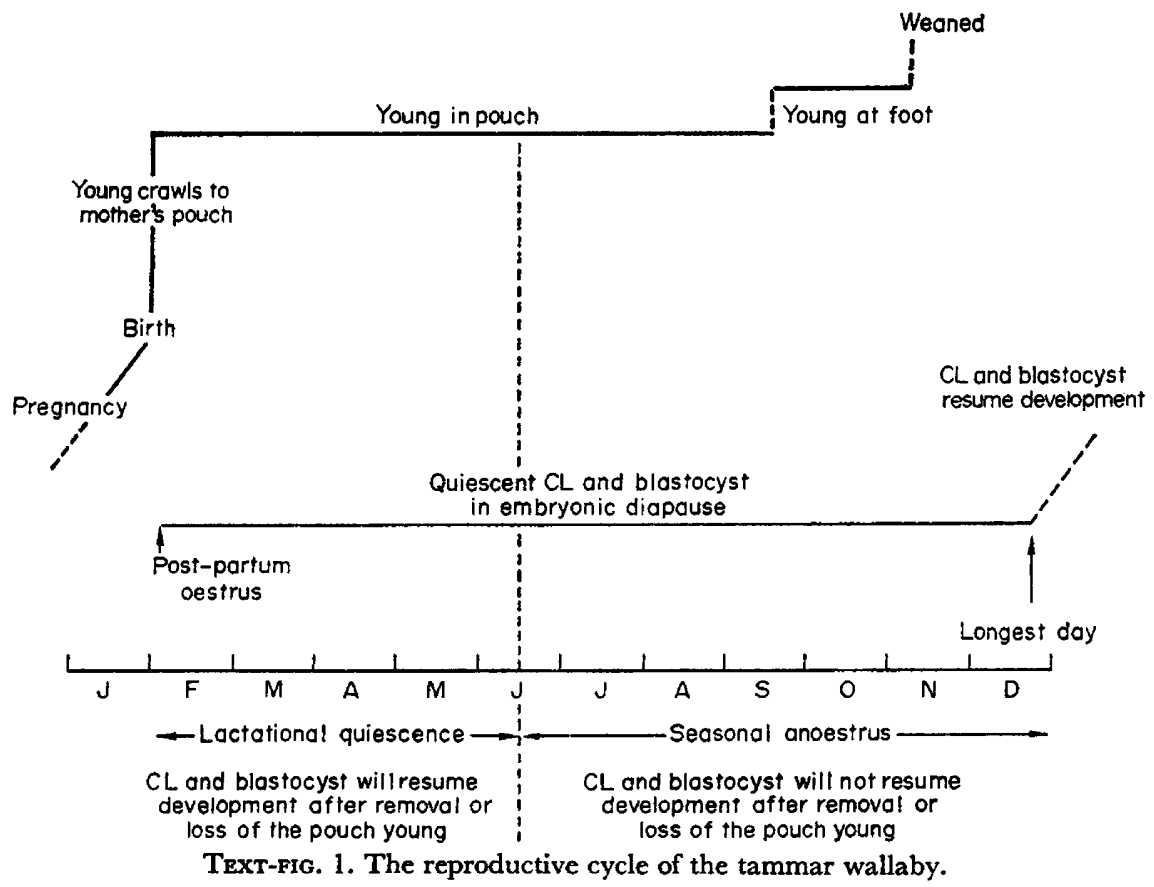

* Present address: M.R.C. Unit of Reproductive Biology, 39 Chalmers Street, Edinburgh. 
spends up to 8 months in the mother's pouch, and during this time the mother is in a state of lactational anoestrus (Berger \& Sharman, 1969). This merges with a seasonal anoestrus between about June and December. There is a postpartum oestrus 12 to $24 \mathrm{hr}$ after birth, and ovulation follows 12 to $24 \mathrm{hr}$ later. If conception occurs at this time the embryo only develops as far as a unilaminar blastocyst with approximately eighty cells (Smith \& Sharman, 1969) before entering an 11-month phase of quiescence termed embryonic diapause (TyndaleBiscoe, 1963) when it remains free in the lumen of the uterus. The corpus luteum of diapause also remains small and inactive throughout this period. Loss or removal of the pouch young between January and June causes the quiescent corpus luteum and the blastocyst to resume development and the new young is born about 27 days later (Berger, 1970). Implantation occurs between Days 17 and 20 of this 27 -day pregnancy. In contrast to this, removal of pouch young during the seasonal anoestrus between June and December has no effect on the quiescent corpus luteum and blastocyst, and development is not resumed until late December, presumably in response to an environmental stimulus, such as the longest day. Berger (1970) has shown that translocation of wallabies to the northern hemisphere results in a reversal of breeding seasonality, implicating photoperiod as a factor in this precise resumption of embryonic development at the start of the breeding season.

The purpose of this study was to examine, by means of hypophysectomy and radioimmunoassay of total plasma gonadotrophin, the rôle of the pituitary gland during pregnancy and embryonic diapause.

\section{MATERIALS AND METHODS}

\section{Animals}

Tammar wallabies were caught at night, with spotlight and nets from a moving vehicle, on Kangaroo Island, South Australia. They were kept in large well-grassed enclosures at the C.S.I.R.O. Division of Wildlife Research, Canberra. The animals used in experiments involving hypophysectomy or sham hypophysectomy were transferred to the Zoology Department, Australian National University, where they were caged and maintained for 6 days before operation, being subjected to the same routine of feeding, handling and bleeding that they would experience after hypophysectomy. This familiarization was intended to reduce maintenance stress after the operation.

\section{Dating of pregnancies}

Removal of the pouch young from the female tammar wallaby between January and June causes resumption of development of the quiescent corpus luteum and blastocyst; a new young is born 27 days later. In this study the time of start of 'pregnancy' was taken as the time of removal of pouch young.

\section{Hypophysectomy}

Wallabies were anaesthetized with $30 \mathrm{mg}$ Nembutal (Abbott)/kg body wt and anaesthesia was maintained during the operation using a cannula in the ear vein; respiration rate was used to assess the depth of anaesthesia. The lower 
jaw and neck were shaved and a tracheal cannula was inserted through a small transverse incision between two tracheal rings to assure uninterrupted breathing during the operation. The wallaby was placed on its back on an operating table, its head held in a simple frame with the mouth at half gape. A 1.5-cm skin incision was made by the left mandible immediately anterior to the submaxillary salivary gland. A Ward's malleable lamp was placed in the mouth and with the aid of this transillumination a narrow approach was made around the side of the buccal cavity and through the nasopharynx until the sphenoid bone was exposed. All blood vessels and nerves were carefully avoided and the approach was made by separating the muscle blocks and connective tissue so that no cutting of tissues was necessary.

The lamp in the animal's mouth was now adjusted to shine through the soft palate and illuminate the sphenoid bone, through which a median hole, $8 \times 4 \mathrm{~mm}$, was slowly drilled immediately above the pituitary. The dura mater was exposed over the whole area of the sella turcica. The dura mater and the connective tissue capsule of the pituitary were then cut through with a No. 11 scalpel blade masked to the correct depth, carefully avoiding the branches of the internal carotid arteries which run ventrolaterally between the dura mater and the pituitary at this point. The pituitary was removed by suction and the hole plugged with Gelfoam (Upjohn) and Horsley's bone wax (Martindale Samoor). After blowing air through the trachea and nasopharynx to expel any blood and saliva, the skin incisions were closed with surgical clamps.

Wallabies usually recovered rapidly after the operation and survived in apparent good health up to 120 days, the longest period required for the experiments carried out in this study. Although animals appeared quite normal within a few hours of hypophysectomy, the essential indication of return to health was a resumption of normal feeding and drinking. An adrenal crisis occurred within 2 days of the operation and when this was severe it could be treated with a single injection of $2 \mathrm{mg}$ hydrocortisone $/ \mathrm{kg}$ body wt. Apart from the provision of $0.9 \%$ saline no further therapy was required.

The sham hypophysectomy procedure was identical to that described above except that the pituitary was not sucked out. Completeness of hypophysectomy was checked routinely in three ways. At operation the removed pituitary was carefully examined for completeness; it was usually removed whole and with practice it was obvious if any parts were missing. The wide surgical approach through the sphenoid allowed examination of the sella turcica for any pituitary remnants before the operation was completed. At autopsy the hypothalamus and entire sella turcica were carefully inspected, serially sectioned and examined for pituitary fragments. Of the seventy-five wallabies hypophysectomized, one was found to have a pituitary remnant when the sella was sectioned and this animal was already known to be incompletely hypophysectomized from the checks made at the time of operation.

\section{Radioimmunoassay of gonadotrophin}

The assay was developed for extracts of wallaby pituitary glands after it was found that wallaby gonadotrophins would not cross-react with NIH-ovine FSH 
and cross-reacted only slightly with NIH-ovine LH. As there is no knowledge of marsupial gonadotrophin chemistry and only small quantities of wallaby pituitary material could be obtained, assays were developed for 'total gonadotrophin' without further fractionation to individual gonadotrophins for this initial study. No fully specific plasma FSH assay had at the time been reported for any species and it was surmised that a total gonadotrophin approach would yield a predominantly $\mathrm{LH}$ assay.

Wallaby pituitary glands were extracted by the method that Hartree (1966) developed for human pituitaries and has since applied successfully to gonadotrophin extraction of horse, chicken and dog pituitary glands (Hartree, Mills, Welch \& Thomas, 1968; Hartree \& Cunningham, 1969; Hartree, Bell, Christie \& Kirkham, 1972). Antisera raised in rabbits to the wallaby gonadotrophin extracts showed an inhibitory activity in an adapted mouse uterine-weight augmentation bioassay. The double-antibody radioimmunoassay was based on that of Odell, Ross \& Rayford (1966) with turkey antirabbit $\gamma$-globulin employed as precipitating antibody. The assay was tested and found specific for wallaby gonadotrophin since it did not cross-react with NIH-LH, FSH or prolactin, or with red kangaroo gonadotrophin or sheep gonadotrophin extracted by the same method. The sensitive range of the assay was from 1 to $40 \mathrm{ng}$ wallaby gonadotrophin.

Blood samples taken from six adult female wallabies over a period of 60 days from February to April, during which each animal experienced a pregnancy and an oestrous cycle, were measured for plasma gonadotrophin. Samples were taken every day between 08.00 and 10.00 hours for 6 days around oestrus and for 2 days before and 4 days following the removal of pouch young. Over the remainder of the period samples were taken every 4 days. Samples were taken from the same animals in August and October to examine whether gonadotrophin levels varied during seasonal anoestrus. Samples were taken from all hypophysectomized wallabies.

\section{RESULTS}

\section{Hypophysectomy during pregnancy}

Groups of three wallabies were hypophysectomized or sham-hypophysectomized at the time of removal of pouch young and on Days 4, 8, 12, 16 and 20 of pregnancy induced by removal of pouch young. Autopsies were carried out on Days 20 and 24 of pregnancy, and 2 days after expected birth. There was no disruption of implantation or of the normal course of pregnancy, but the group hypophysectomized on Day 4 showed a 2 to 4 day retardation of embryonic growth at autopsy on Day 20. Parturition did not take place after hypophysectomy: at autopsy 2 days after expected birth there was always a dead full-term fetus in the uterus. The development of the corpus luteum of pregnancy also proceeded normally but ovarian follicular growth and oestrous changes in the lateral and median vaginae and in the pouch were arrested from the time of hypophysectomy. In contrast, all sham hypophysectomized control animals gave birth on the expected dates and had a normal post-partum oestrus and ovulation. Therefore the pituitary gland was not 
necessary for any stage of pregnancy following the removal of pouch young, but was required for parturition.

\section{Hypophysectomy during lactation quiescence}

Three wallabies carrying pouch young were hypophysectomized in March and April during lactational anoestrus but before seasonal anoestrus. Lactation ceased within 2 days: no milk could be expressed from the teats and the pouch young began to lose weight rapidly although they remained on the teats for a further 2 to 5 days. At autopsy 24 days after hypophysectomy all were carrying a live embryo in the uterus in terminal stages of pregnancy. A control group of three sham-hypophysectomized wallabies continued to lactate, did not lose their pouch young and showed no resumption of development of quiescent corpora lutea or blastocysts. Thus the resumption of corpus luteum and blastocyst growth after removal of pouch young is not dependent on pituitary stimulation.

\section{Hypophysectomy during seasonal anoestrus}

In the normal cycle of the wallaby, removal of pouch young during seasonal anoestrus does not terminate embryonic diapause, which continues until the following December. Six lactating and seven non-lactating wallabies were therefore hypophysectomized during seasonal anoestrus. In all of them embryonic diapause was terminated and the quiescent corpora lutea and blastocysts resumed development. In the six lactating animals, lactation stopped within 2 days of hypophysectomy, but at autopsy on Day 20 of pregnancy embryonic growth was found to be much retarded; embryos were at the stages found normally between Days 6 to 14 of pregnancy. The corpora lutea were correspondingly small. The non-lactating wallabies showed normal-sized corpora lutea and embryos at autopsy on Day 20 of pregnancy.

In five sham-hypophysectomized control animals there was no disruption of the normal cycle: lactation was not interrupted and embryonic diapause continued. Therefore although all the anoestrous wallabies showed a resumption of embryonic growth after hypophysectomy, the ensuing pregnancy was more successful in those that were not lactating at the time of operation.

\section{Plasma gonadotrophin levels}

Plasma levels of gonadotrophins in six intact female wallabies were basal at 2 to $5 \mathrm{ng} / \mathrm{ml}$ throughout pregnancy but at oestrus showed peaks of 11 to 18 $\mathrm{ng} / \mathrm{ml}$. There was no rise in plasma gonadotrophin in the days following removal of pouch young when the corpus luteum and blastocyst resumed development. The basal plasma gonadotrophin level was not significantly different in the same animals during lactation quiescence and during the seasonal anoestrus. Control plasma taken from every hypophysectomized wallaby gave undetectable values, while plasma levels in six ovariectomized wallabies rose to 5 to $7 \mathrm{ng} / \mathrm{ml}$ within 2 months of the operation.

\section{DISCUSSION}

This study was based on knowledge of eutherian species (reviewed by Amoroso \& Porter, 1966; Rowlands \& Parkes, 1966; Deanesly, 1966) from which it was 
expected that hypophysectomy before implantation would cause immediate termination of pregnancy but that hypophysectomy after implantation might not. It was expected also that embryonic diapause would be produced by an inactive corpus luteum consequent upon insufficient pituitary stimulation. The results were completely at variance with these hypotheses. They clearly demonstrate that the pituitary gland is required neither for activation or maintenance of the corpus luteum of pregnancy, nor for the resumption of blastocyst growth and maintenance of pregnancy in the wallaby. The pituitary must, however, be present for successful parturition to occur. The results strongly suggest that embryonic diapause in this species is controlled by a tonic pituitary inhibition of the corpus luteum (Hearn, 1973).

Although hypophysectomy on the 4th day of pregnancy slightly retarded embryonic development, no such effect was evident when the operation was performed on the first day, at the time of removal of the pouch young. In the latter case presumably the animals were over the operation stress before the blastocyst resumed growth; the retardation effect seen in the Day-4 group was probably due to operational stress or its metabolic consequences for which sham hypophysectomy could not act as a full control. The corpus luteum of pregnancy appeared unaffected by hypophysectomy in the wallaby.

The radioimmunoassay results provided corroborative evidence that there is no marked plasma gonadotrophin increase above basal levels during pregnancy in the wallaby, even after removal of the pouch young, when a surge of gonadotrophin might have been expected to coincide with the resumption of embryonic development.

The pregnancy that follows removal of pouch young depends in its early stages on the corpus luteum; once the corpus luteum resumes development that of the uterus and embryo follows, and all three can be regarded as an autonomous unit over this period. Tyndale-Biscoe (1970) has shown by ovariectomy and blastocyst transfer that by Day 8 of pregnancy the uterus itself is competent to nourish an embryo to full term without further ovarian stimulation, but that pregnancy terminates if ovariectomy is performed before Day 8. The focal point of interest is the factor controlling resumption of development of the corpus luteum, and these experiments suggest that the corpus luteum is activated by the removal of a restraining influence from the pituitary. Lactational inhibition is well known in many eutherian and marsupial species, but cannot explain the resumption of embryonic development seen in non-lactating wallabies hypophysectomized during seasonal anoestrus. The only known inhibitor of the corpus luteum in a marsupial is oxytocin, which can apparently prolong diapause for a short time after removal of pouch young from the lactating red kangaroo (Sharman, 1965). If oxytocin is the inhibitory agent in the wallaby, however, a prolonged secretion of oxytocin would be required in the absence of lactation during seasonal anoestrus; this seems highly unlikely.

This investigation of the mechanism controlling embryonic diapause in a marsupial emphasizes that different species have developed radically different ways of achieving the same objective, namely an environmental control over the time of implantation. The marsupial evidence points strongly to a dominant 
pituitary inhibition of the corpus luteum; perhaps we should be alerted to the possibility of a similar mechanism in some eutherian species.

\section{AGKNOWLEDGMENTS}

I am most grateful to Dr C. H. Tyndale-Biscoe for his advice throughout this study and to Dr R. V. Short for his critical reading of the manuscript.

\section{REFERENGES}

Amoroso, E. G. \& Porter, D. G. (1966) Anterior pituitary function in pregnancy. In The Pituitary Gland. Eds. G. W. Harris and B. T. Donovan. Butterworths, London.

Berger, P. J. (1970) The reproductive biology of the tammar wallaby, Macropus eugenii. Ph.D. thesis, Tulane University.

Berger, P. J. \& Sharman, G. B. (1969) Progesterone-induced development of dormant blastocysts in the tammar wallaby, Macropus eugenii Desmarest; Marsupialia. F. Reprod. Fert. 20, 201.

Deanessy, R. (1966) The endocrinology of pregnancy and foetal life. In Marshall's Physiology of Reproduction, Vol. 3, pp. 891-1063. Ed. A. S. Parkes. Longmans Green, London.

HARTREe, A. S. (1966) Separation and partial purification of the protein hormones from human pituitary glands. Biochem. F. 100, 754 .

Hartree, A. S., Bell, E. T., Ghristie, D. W. \& Kirkham, K. E. (1972) Partial purification of dog pituitary glycoprotein hormones. F. Endocr. 52, 403.

Hartree, A. S. \& Cunningham, F. J. (1969) Purification of chicken pituitary FSH and LH. F. Endocr. 43, 609.

Hartree, A. S., Milis, J. B., Welch, R. A. S. \& Thomas, M. (1968) Fractionation of protein hormones from horse pituitary glands. $\mathcal{F}$. Reprod. Fert. 17, 291.

Hearn, J. P. (1973) Pituitary inhibition of pregnancy. Nature, Lond. 241, 207.

Odell, W. D., Ross, G. T. \& RAyford, P. L. (1966) Radioimmunoassay for human luteinising hormone. Metabolism, 15, 287.

Rowlands, I. W. \& Parkes, A. S. (1966) Hypophysectomy and the gonadotrophins. In Marshall's Physiology of Reproduction, Vol. 3, pp. 26-146. Ed. A. S. Parkes. Longmans Green, London.

Sharman, G. B. (1965) The effects of the suckling stimulus and oxytocin injection on the corpus luteum of delayed implantation in the red kangaroo. Excerpta med. 83, 669.

Sharman, G. B., Calaby, J. H. \& Poole, W. E. (1966) Patterns of reproduction in female diprotodont marsupials. Symp. zool. Soc. Lond. 15, 205.

Smith, M. J. \& Sharman, G. B. (1969) Development of dormant blastocysts induced by oestrogen in the ovariectomised marsupial Macropus eugenii. Aust. J. biol. Sci. 22, 171.

TyNDALE-Biscoe, C. H. (1963) The role of the corpus luteum in delayed implantation in marsupials. In Delayed Implantation, pp. 15-32. Ed. A. G. Enders. Chicago University Press.

Tyndale-Biscos, G. H. (1970) Resumption of development by quiescent blastocysts transferred to primed, ovariectomized recipients in the marsupial, Macropus eugenii. F. Reprod. Fert. 23, 25.

Tyndale-Biscoe, G. H. (1973) The Life of Marsupials, Edward Arnold, London. 